faithfully carried out, and the patient was now able to stand and walk erect, and in the course of another year, the speaker said, his condition would probably be entirely normal. At present he was able, by voluntary muscle action, to antagonize every tic contraction, but this balancing activity had not yet becn developed to the point of being automatic, as it normally should bc. The speaker then explained briefly the psychical regime which was part of the psychophysical therapeutics, which included the development of the patient's intellectual and moral strength, the training of his powers of initiative and inhibition, regularity and system in habits, etc.

The physical training consisted in medical gymnastics, starting from an analysis of the tic-attitude into its components, the elementary musclespasms. Each of these tic-elements had to receive a remedying antagonistic muscle-exercise. At the ontset, the patient was not able to make the antagonizer of a tic-muscle contract by itself. This required a series of assisted exercises. In these, the patient was helped by an assisting force, outside of his own muscular effort, to overcome the tic-spasm. The percentage of efficiency of the patient's own efforts would show increase according to their repetition, and the assisting force was correspondingly diminished. Finally, the assisted exercises became unnecessary and were replaced by unassisted ones. The first land mark of progress was the ability to overcome every tic element by its antagonistic action, and every tic attitude by its counter attitude; the second, identical with re-establishment of the normal condition, would be reached when these voluntary antagonistic actions, by untiring exercise, had become automatic.

Dr. William M. Leszynsky, who had presented the same patient at a meeting of this society several years ago, said that Dr. Kruna was certainly to be congratulated upon the brilliant restult he had obtained from his psychophysical method of treatment. When Dr. Leszynsky had, him under his care there was some improvement after a similar method of treatment in the hospital, but within five montns he relapsed into his original condition. He was then sent to the Hospital for the Ruptured and Crippled, where he was fitted with various kinds of apparatus, and several muscles tenotomized without relief. He was finally transferred to the Montefiore Home. The diagnosis of the case at that time was hysteria, and his physical condition was associated with a peculiar mental attitude which seemed to point to some form of degeneracy.

\title{
TWO CASES OF CEREBRAL APOPLEXY, WITH UNUSUAL DISTRIBUTION OF THE SYMPTOMS
}

By Dr. I. Abrahamson

The first patient was a man, 64 years old, a native of Russia, who was admitted to Mt. Sinai Hospital on April 6, 1908. He had long been addicted to the use of alcohol and cigarettes, both to excess. For three or four weeks prior to admission he had complained of transient attacks of paresthesia of the left hand and wrist, with numbness and weakness. These attacks, which occurred about once a week, were of a few minutes duration, and upon one occasion were associated with mental confusion.

Without further warning, he had a rather sudden attack of paralysis involving the left face and arm; this was transient in character, lasting 
only a few minutes, and following this, he had a number of similar atattacks. In all of these the left face was involved and his speech was thick, but not lost; these persisted for 24 hours and the last left patient paralyzed. There was never a loss of consciousness; no sphincteric trouble nor vomiting. He had never had severe headaches. Examination showed a facio-brachial partial hemiplegia; leg not at all involved; cause, thrombosis of the second branch of the Sylvian artery; arteriosclerotic in origin.

The second patient shown by Dr. Abrahamson was a woman, 49 years old, a native of Scotland, who was admitted to the Mt. Sinai Hospital on April 19, rgo8. The history relating to her present illness dated back three days prior to her admission, when her husband struck her with his fist at the nape of the neck. She fell from the force of the biow, but arose at once and walked to the bed, where she fell again, but succeeded in getting into bed. Shortly afterwards, the fingers of her left hand began to feel numb and she could not move the left arm. The paralysis gradually extended to the face and leg, and her speech became thick. Denies she suffered from unconsciousness. There was no history of vomiting; no loss of control of the sphincters. At the present time there was flattening of the left side of the face, and the tongue was deviated slightly to the left: motor weakness left upper extremity with exaggeration of the deep reflexes; only slight involvement of left lower extremity, etc., i. e., a facio-brachial cerebral palsy. Here the cause was a hemorrhage, rather small, in the neighborhood of the internal capsule.

Both patients showed an unusual amount of implication of the upper facial musculature.

Incomplete hemiplegias are always of interest, especially traumatic hemorrhages into the brain substance.

The President, Dr. B. Sachs, who had both of these patients under his observation at Mt. Sinai Hospital, said they were interesting examples of facio-brachial paralysis, both occurring in the manner of an apoplexy. The woman developed this peculiar form of hemiplegia after receiving a blow behind the ear, and the disability was limited to the face and arm.

Dr. Hunt said he had seen several cases of this type, and had one under his observation at the present time, with almost complete monoplegia of the left arm, only slight weakness of the left face and no involvement of the leg at all. The onset in this case pointed to a cerebral lesion, in all probability in the neighborhood of the cortex. This patient had had a number of convulsive seizures of the Jacksonian type. There were no sensory disturbances and the lesion had not progressed.

During the recent epidemic of poliomyelitis, Dr. Hunt said he saw an old woman, very much emaciated, with a complete flaccid paralysis of the left upper extremity. There was a total absence of any signs pointing to a hemiplegia and he was inclined to regard it either as a spinal hemorrhage localized in the anterior horn, or as an unusual form of poliomyelitis. In the course of a few weeks the flaccidity gave way to a mild increase in the reflexes with slight rigidity. In this case there was no involvement of the face or leg, nor were there any sensory disturbances. She gave no history of headaches, although she had suffered from transient attacks of vertigo during the last year. The case was apparently one of cerebral monoplegia with no trace of involvement of the face or leg, and without irritative symptoms pointing to the cerebral cortex.

Dr. Smith Ely Jelliffe said he had seen a number of cases of faciobrachial paralysis--perhaps half a dozen during the past year. In most 
of the cases the histories were similar to those given by Dr. Abrahamson, the onset being mild and the symptoms chiefly limited to the face and upper extremity, with slight impairment, in some instances, of the lower extremity. Through the courtesy of Dr. Janeway, the speaker said, he recently saw a case presenting this, with other features on the oposite side, at the City Hospital, in which the attack was extrenely severe. The patient was comatose for a week, but the residual effects of the apoplectic seizure were very slight, being limited on the riglit side to a paralysis of the arm and lower face. There was no increase in the knee or ankle reflexes; no clonus; no Babinski on the right side. On the opposite side, however, there was a persistent chorea-like tremor, including the face, arm, and leg, with Babinski, clonus, and increased knee jerks, but no paralysis. The tremor was of the semi-intentional type, averaging 180 to the minute. There were marked sensory disturbances on the right side, especially to pain and to temperature. Deep sensibility was affected, but not light touch. Sensory disturbances were absent on the left side.

Dr. William Hirsch thought the main reason that cerebral monoplcgia was regarded as unustual or rare was that we always had in mind that the most frequent hemorrhages occurred in the internal capsule, and were apt to overook the fact that those forms of paralysis due to arteriosclerosis, syphilis or thrombosis were more likely to give rise to a diplegia or a monoplegia rather than a complete hemiplegia. In a case of cerebral arteriosclerosis seen comparatively recently the only manifestations were an aphonia, with very slight paralysis of the face. In another case, the only symptom was alexia. A clear distinction should be drawn between these two classes of cases; in one, complete hemiplegia was the rule, while in the other, where we had to deal with vascular changes, a complete hemiplegia was the exception, rather than the rule.

Dr. Leszynsky said he had seen a number of cases of cerebral hemorrhage in which only the face and arm were involved, but, as a rule, upon investigation one usually learned that the entire side was affected at first and that the leg recovered within a few hours or days. Sucl cases were not at all uncommon in his experience.

Dr. Sachs said that in the internal capsule the fibers were so close together that it was almost inconceivable that a hemorrhage there would involve the arm fibers and leave those of the leg exempt. It would perhaps become necessary at some future date to change our present views regarding the arrangement of fibers in the internal capsule.

\section{DISCUSSION ON THE PRESENT-DAY LIMITATION OF THE CONCEPTION OF PARANOIA}

\section{By Dr. M. S. Gregory}

In opening this discussion, the speaker said that he would not attempt a complete historical résumé of the paranoia problem, interesting and instructive though it might be, and would consider only those later day conceptions which were still subjects of controversy. He would make no pretense of trying to solve the paranoia problem. It was well known that the origins of paranoid delusion fornation were extremely complicated, and it was not his intention to attempt a complete psychological analysis, notwithstanding its great importance, and in spite of the fact that it was 